\title{
Time Course of Coagulation and Fibrinolytic Parameters in Patients with Traumatic Brain Injury
}

\section{R. Nakae, S. Yokobori, Y. Takayama, T. Kanaya, Y. Fujiki, Y. Igarashi, G. Suzuki, Y. Naoe, A. Fuse, and H. Yokota Department of Emergency and Critical Care Medicine, Nippon Medical School, Tokyo, Japan}

\section{Introduction}

Traumatic brain injury (TBI) has long been associated with coagulopathy; however, the time course of coagulation/fibrinolytic parameters in the acute phase of TBI remains unclear. The purpose of the study was to analyze the time course of coagulation/fibrinolytic parameters in the acute phase of TBI and to elucidate parameter relationships to prognosis.

\section{Materials and Methods}

- We retrospectively evaluated 234 patients with severe isolated TBI with initial blood samples obtained no more than $1 \mathrm{~h}$ after injury.

- Platelet count, prothrombin time, activated partial thromboplastin time (aPTT), plasma levels of fibrinogen, and D-dimer were measured on arrival and 3, 6, and $12 \mathrm{~h}$ after injury.

- Multivariate logistic regression analysis was performed to identify risk factors for poor prognosis at each time point.

\section{Results}

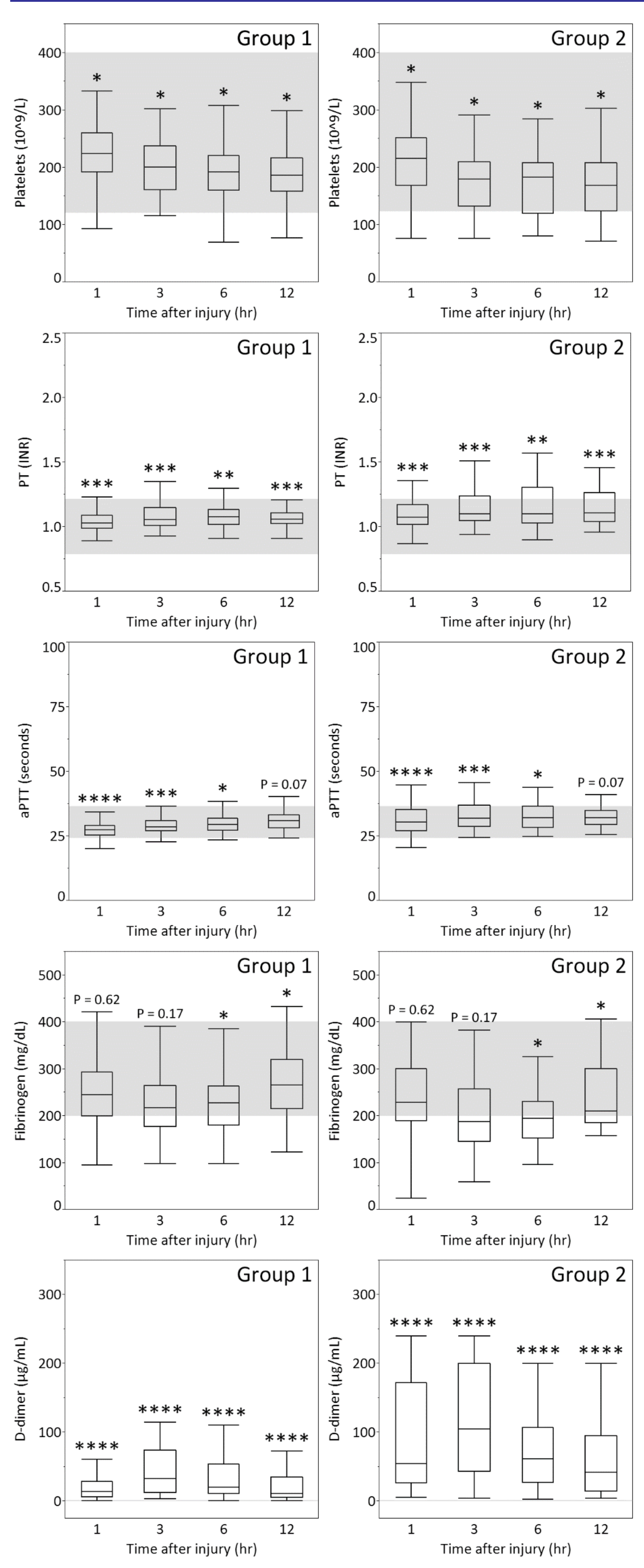

FIGURE. Platelet count, PT, aPTT, and plasma levels of fibrinogen and D-dimer of patients with a good outcome (Group 1; GOS at 3 months = GR/MD) or poor outcome (Group 2; GOS at 3 months = SD/VS/D) on admission and at 3, 6, and $12 \mathrm{~h}$ after TBI.

The most marked difference between group 1 and group 2 at all time points from admission to $12 \mathrm{~h}$ after injury was in the plasma level of D-dimer $\left(p<0.0001\right.$ at all time points). $* p<0.05,{ }^{*} p<0.01, * * * p<0.001, * * * * p<0.0001$

\begin{tabular}{|c|c|c|c|c|c|c|c|c|}
\hline \multirow[b]{2}{*}{ Factor } & \multicolumn{2}{|c|}{ admission } & \multicolumn{2}{|c|}{3 hours after injury } & \multicolumn{2}{|c|}{6 hours after injury } & \multicolumn{2}{|c|}{12 hours after injury } \\
\hline & $\begin{array}{c}\mathrm{OR} \\
(95 \% \mathrm{Cl})\end{array}$ & $\mathrm{p}$ & $\begin{array}{c}\mathrm{OR} \\
(95 \% \mathrm{Cl})\end{array}$ & $\mathrm{p}$ & $\begin{array}{c}\mathrm{OR} \\
(95 \% \mathrm{Cl})\end{array}$ & $\mathrm{p}$ & $\begin{array}{c}\mathrm{OR} \\
(95 \% \mathrm{Cl})\end{array}$ & $\mathrm{p}$ \\
\hline $\begin{array}{c}\text { Platelets } \\
\left(10 \times 10^{9} / \text { L decrements }\right)\end{array}$ & $\begin{array}{c}1.02 \\
(0.96-1.09)\end{array}$ & 0.53 & $\begin{array}{c}1.01 \\
(0.89-1.14)\end{array}$ & 0.89 & $\begin{array}{c}1.01 \\
(0.90-1.14)\end{array}$ & 0.85 & $\begin{array}{c}1.05 \\
(0.94-1.17)\end{array}$ & 0.40 \\
\hline $\begin{array}{c}\text { PT } \\
\text { (0.1 INR increments) }\end{array}$ & $\begin{array}{c}1.00 \\
(0.81-1.29)\end{array}$ & 0.99 & $\begin{array}{c}1.08 \\
(0.69-1.57)\end{array}$ & 0.74 & $\begin{array}{c}1.06 \\
(0.71-1.56)\end{array}$ & 0.77 & $\begin{array}{c}1.34 \\
(0.99-1.99)\end{array}$ & 0.057 \\
\hline $\begin{array}{c}\text { aPTT } \\
\text { (1 second increments) }\end{array}$ & $\begin{array}{c}1.20 \\
(1.07-1.35)\end{array}$ & 0.0011 & $\begin{array}{c}1.23 \\
(1.04-1.50)\end{array}$ & 0.013 & $\begin{array}{c}1.08 \\
(0.93-1.26)\end{array}$ & 0.30 & $\begin{array}{c}1.02 \\
(0.90-1.15)\end{array}$ & 0.72 \\
\hline $\begin{array}{c}\text { Fibrinogen } \\
\text { (10 mg/dL decrements) }\end{array}$ & $\begin{array}{c}1.04 \\
(0.99-1.10)\end{array}$ & 0.11 & $\begin{array}{c}1.04 \\
(0.99-1.10)\end{array}$ & 0.13 & $\begin{array}{c}1.03 \\
(0.94-1.14)\end{array}$ & 0.60 & $\begin{array}{c}1.01 \\
(0.96-1.08)\end{array}$ & 0.64 \\
\hline $\begin{array}{c}\text { D-dimer } \\
(10 \mu \mathrm{g} / \mathrm{mL} \text { increments })\end{array}$ & $\begin{array}{c}1.21 \\
(1.12-1.31)\end{array}$ & $<0.0001$ & $\begin{array}{c}1.15 \\
(1.06-1.27)\end{array}$ & 0.0005 & $\begin{array}{c}1.15 \\
(1.04-1.30)\end{array}$ & 0.005 & $\begin{array}{c}1.22 \\
(1.08-1.41)\end{array}$ & 0.0009 \\
\hline
\end{tabular}

TABLE. Multivariate logistic regression analysis of coagulation-fibrinolytic parameters as independent risk factors for poor prognosis

From admission to $12 \mathrm{~h}$ after injury, an elevated D-dimer level was a significant negative prognostic indicator. An upward trend of aPTT on admission and $3 \mathrm{~h}$ after injury was also a significant negative prognostic indicator. On multivariate logistic regression analysis which included all initial variables, independent risk factors for poor prognosis included older age $(p=0.0005)$, low Glasgow Coma Scale score $(p<0.0001)$, high Abbreviated Injury Score $(p=0.015)$, aPTT $>30.2$ seconds $(p=0.019)$, and elevated D-dimer level $(p=0.0005)$.

\section{Conclusions}

- Coagulation and fibrinolytic parameters undergo dynamic changes during the acute phase of TBI.

- D-dimer is the best coagulation/fibrinolytic parameter to monitor for prediction of outcome.

- Routine measurement of fibrinolytic parameters such as D-dimer to predict the occurrence of coagulopathy and the expected outcome is warranted.

- Future studies should reveal whether early recognition of acute coagulopathy and the prevention of delayed hemostatic disturbances might be associated with improvements in morbidity and mortality in patients with isolated head trauma. 